\title{
Agôn
}

Revue des arts de la scène

$6 \mid 2013$

La Reprise

\section{Jan Fabre au risque de la reprise}

\section{Giulio Boato et Catherine Bouko}

\section{(2) OpenEdition \\ Journals}

\section{Édition électronique}

URL : http://journals.openedition.org/agon/2741

DOI : 10.4000/agon.2741

ISSN : 1961-8581

\section{Éditeur}

Association Agôn

\section{Référence électronique}

Giulio Boato et Catherine Bouko, « Jan Fabre au risque de la reprise », Agôn [En ligne], 6 | 2013, mis en ligne le 23 février 2014, consulté le 22 avril 2019. URL : http://journals.openedition.org/agon/2741 ; DOl : 10.4000/agon.2741

Ce document a été généré automatiquement le 22 avril 2019

Association Agôn et les auteurs des articles 


\title{
Jan Fabre au risque de la reprise
}

\author{
Giulio Boato et Catherine Bouko
}

1 L'heure est à la reprise. Les productions remontées par les créateurs originaux abondent aujourd'hui sur les scènes internationales : Einstein on the Beach de Lucinda Childs, Philip Glass et Bob Wilson en 2012 , auquel la revue Agôn a consacré un numéro, ou le cycle de performances The Artist is Present par Marina Abramovic au MoMA en 2010, sont quelques arbres emblématiques d'une forêt dense et protéiforme.

2 Si ce phénomène se cristallise particulièrement outre-Atlantique, il se développe également en Europe. Le cas particulier de la Belgique néerlandophone en témoigne: Anne Teresa De Keersmaeker reprend régulièrement Rosas danst Rosas (1983) depuis 2009, Fase (1984) depuis 2008 et Elena's aria (1984) depuis 2011 ; en 2013, Wim Vandekeybus part en tournée mondiale avec son premier spectacle What The Body Does Not Remember (1987); en 2012, Jan Fabre reprend quant à lui C'est du Théâtre comme c'était à espérer et à prévoir (1982) et Le Pouvoir des folies théâtrales (1984).

3 Le contexte spectaculaire contemporain favoriserait cette posture rétrospective. Dès 1982, Richard Schechner soulignait la fin du "jaillissement d'énergie expérimentale ${ }^{2}$ propre à l'avant-garde : «Bien que prévisible, l'arrêt partiel - voire complet - de cette activité (les expérimentations, le dépassement des limites et des conventions, l'action politique, la remise en question, la multiplicité des possibilités de mise en scène, le partage de l'essentiel de l'autorité créatrice) nous a pris au dépourvu. [...] Un big bang a laissé place à l'entropie. ${ }^{3}$ "

4 Tel un phénomène en escaliers, nous rencontrons parfois une approche nostalgique semblable de la production spectaculaire flamande des années quatre-vingt. Pour le dramaturge Erwin Jans, «nous travaillons encore toujours dans l'ombre du renouvellement qui a caractérisé les années quatre-vingt. ${ }^{4}$ " Jan Fabre lui-même exprime ce point de vue lorsqu'il affirme que ses spectacles témoignent toujours d'une "radicalité sans équivalent dans le théâtre actuel ${ }^{5} »-$ propos qui ulcèrent par ailleurs ses détracteurs.

5 Schechner ${ }^{6}$ souligne combien cette posture d'autoreprésentation, « entre narcissisme et générosité $»^{7}$, se nourrit d'une double revendication : 1) il y a des classiques de l'avant- 
garde, appartenant désormais au répertoire et à l'histoire du théâtre ;2) ces spectacles touchent les nouvelles générations de spectateurs, dans leur forme originale.

6 Cette double revendication ne va cependant pas de soi et engendre une série de paradoxes, de contradictions et de risques autour de la pratique des reprises. La reprise tend en effet à transformer un geste d'énonciation, au potentiel subversif, en énoncé, qui devient un objet de contemplation nostalgico-fascinée. Par quelles modalités des spectacles inscrits à la marge lors de leur création peuvent-ils devenir des œuvres de répertoire et toucher le public d'aujourd'hui ? Frédéric Maurin ${ }^{8}$ souligne le risque de dévitaliser l'œuvre en la réifiant et de la transformer en simulacre à partir du moment où la valeur d'échange supplante la valeur d'usage. David Savran insiste lui aussi sur l'ambiguïté de la démarche de reprise, source de nombreuses contradictions : «Si la première avantgarde [...] représentait une protestation contre la chosification de l'art, ses vestiges sacrés actuellement représentent une sorte d'hommage à la culture médiatisée, une tentative d'à la fois mépriser et embrasser la sphère commerciale. ${ }^{9}$ " Les reprises de Jan Fabre témoignent-elles de ce processus d'institutionnalisation de l'avant-garde, qui impliquerait un changement dans la nature du geste artistique?

7 Dans cet article, nous examinerons la délicate entreprise des reprises fabriennes de C'est du Théâtre comme c'était à espérer et à prévoir et du Pouvoir des folies théâtrales selon trois angles. Premièrement, nous aborderons les processus de recréation de ces deux spectacles en traitant les questions de la documentation et de la physicalité des performers. Deuxièmement, nous traiterons de l'authenticité de la présence scénique. Enfin, nous évoquerons la question des attentes des spectateurs aujourd'hui.

\section{Quelques repères sur les spectacles}

En 1982 est présenté à Bruxelles C'est du théâtre comme c'était à espérer et à prévoir, le second spectacle théâtral de Jan Fabre. Le metteur en scène sélectionne huit performers parmi les dizaines qui s'étaient présentés aux auditions. Seulement l'une d'entre eux est actrice professionnelle : «je les ai choisis pour leur univers personnel, leur mentalité. [...] Et si je travaille avec des amateurs, c'est qu'ils ne sont pas déformés par des tics ou des recettes théâtrales ${ }^{10}$.» Au terme de la période de répétition d'une durée de six mois sont obtenues quatorze heures de matière, qui seront réduites à huit.

Le spectacle ne suit pas une continuité narrative. Durant les huit heures se succèdent dixneuf scènes, chacune étant auto-conclusive comme un épisode à part entière. Les scènes sont néanmoins reliées entre elles selon divers critères: associations thématiques (différentes déclinaisons du même thème-argument), associations formelles (objets et éléments visuels qui sont réutilisés, configurations spatiales, sonores ou lumineuses parallèles), ou critères structurels (la structure du spectacle entier est construite selon un principe de symétrie : certaines scènes sont le miroir d'autres, parfois très éloignées dans le temps). 
C'est du Théâtre comme c'était à espérer et à prévoir de Jan Fabre (reprise)

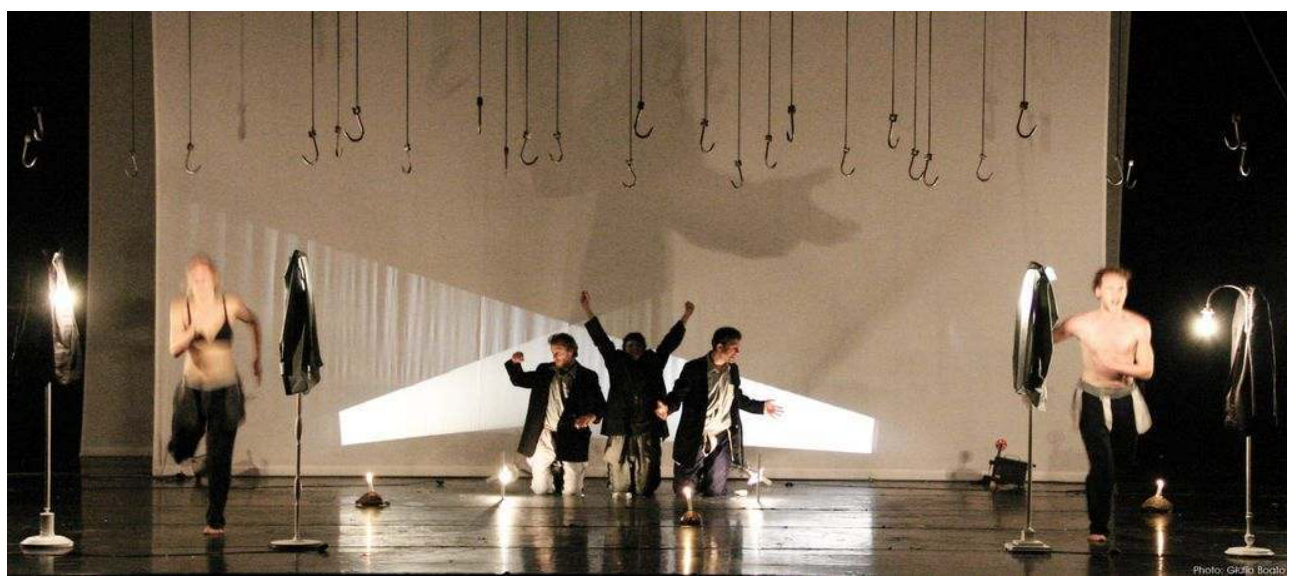

(c) Giulio Boato

10 Le public assiste à une mise à exécution d'actions simples accomplies en temps réel (transporter des sièges, courir, se raser, fumer, etc.). Les mouvements dans l'espace sont pour la plupart exécutés lentement et avec grande attention; le temps est un élément d'une importance fondamentale, tant pour les acteurs que pour les spectateurs. Les fragments textuels sont répétés de nombreuses fois, devenant ainsi un élément sonore au-delà de toute fonction signifiante.

11 C'est du théâtre comme c'était à espérer et à prévoir est un spectacle entièrement dédié à la tentative d'unir l'art contemporain et le théâtre, en intégrant à la représentation l'art de l'installation et la performance. Les thèmes abordés et les matériaux utilisés en scène sont pour la plupart des citations d'œuvres d'autres plasticiens du vingtième siècle, maîtres spirituels du metteur en scène ${ }^{11}$. Les uniques éléments scénographiques permanents sont un écran de projection en fond de scène (faisant aussi office de coulisse pour les entrées et sorties des acteurs) et vingt-neuf crochets de boucher pendus au plafond. L'illumination est produite par onze lampes sur pieds en fer peintes en blanc, chacune légèrement différente de l'autre. L'apport musical est constitué des compositions au synthétiseur créées par Guy D. Drieghe.

12 Après une série de premières représentations de C'est du Théâtre... en Belgique, par ailleurs négativement appréciées par le public et la critique locaux, la compagnie de Fabre est invitée au Mickery Theater d'Amsterdam, important lieu de spectacles dans lequel ont travaillé plusieurs grandes figures du théâtre contemporain, comme Bob Wilson, Richard Foreman ou Tadeusz Kantor. C'est des Pays-Bas que la tournée prend son envol en direction de différents festivals européens. L'atterrissage se fait de nouveau outreAtlantique. La critique demeure divisée - caractéristique de la réception du travail de Fabre - mais la mèche est dorénavant incandescente. Le spectacle commence à faire parler de lui et diverses personnalités du milieu (on peut citer Merce Cunningham, Patrice Chéreau, etc.) assistent aux représentations : « on s'ennuie, on s'endort. Tout n'est pas parfait, loin de là. Les tics du plasticien ayant fait ses classes dans la "performance" sont légion, les fausses provocations aussi. Mais le souvenir de cette nuit de bout en bout excessive balaie tout ${ }^{12}$. »

13 Grâce au succès obtenu avec C'est du théâtre..., Fabre trouve des producteurs pour le projet suivant. Quelques centaines d'acteurs et de danseurs se présentent aux auditions pour Le 
Pouvoir des folies théâtrales, mais les quinze sélectionnés (tous ont entre vingt et trente ans), sont à nouveau des non-professionnels. Le choix est plus ample, mais ce que Fabre nomme la « rage » ou la « passion » reste le critère de recherche numéro un : « when I have to choose between anger and technique, I will always choose the one who has anger ${ }^{13}$.» De nouveau, la compagnie se retire dans un petit centre et travaille intensément durant quatre mois, avec les mêmes modalités que pour les précédentes sessions de répétitions (un jour de pause pour neuf jours de travail, vie commune des performers et du metteur en scène).

Le Pouvoir des folies théâtrales reprend de nombreuses caractéristiques du spectacle précédent (actions concrètes en temps réel) pour le mener au résultat contraire: l'esthétisme et le maniérisme d'une exaltation de théâtralité. Le spectacle propose ambitieusement de recouvrir l'histoire du théâtre moderne occidental, de Richard Wagner à Jan Fabre. Les dialogues répétés dans certaines scènes sont composés uniquement de noms de metteurs en scène, compositeurs, chorégraphes, accompagnés de dates et de titres de leurs spectacles les plus importants. Le Pouvoir des folies théâtrales se positionne en hommage et en critique du théâtre bourgeois, de la fiction scénique et des conventions théâtrales, portées à la scène à travers le filtre des récits fantastiques, parmi lesquels Le roi grenouille, Les habits neufs de l'empereur et La belle au bois dormant. Le fil rouge du spectacle (construit à partir de tableaux scéniques indépendants qui se superposent, à l'instar de C'est du théâtre...) est constitué par les figures des deux empereurs nus qui habitent la scène telles des statues vivantes, durant la quasi-totalité du spectacle.

Le Pouvoir des folies théâtrales de Jan Fabre (reprise)

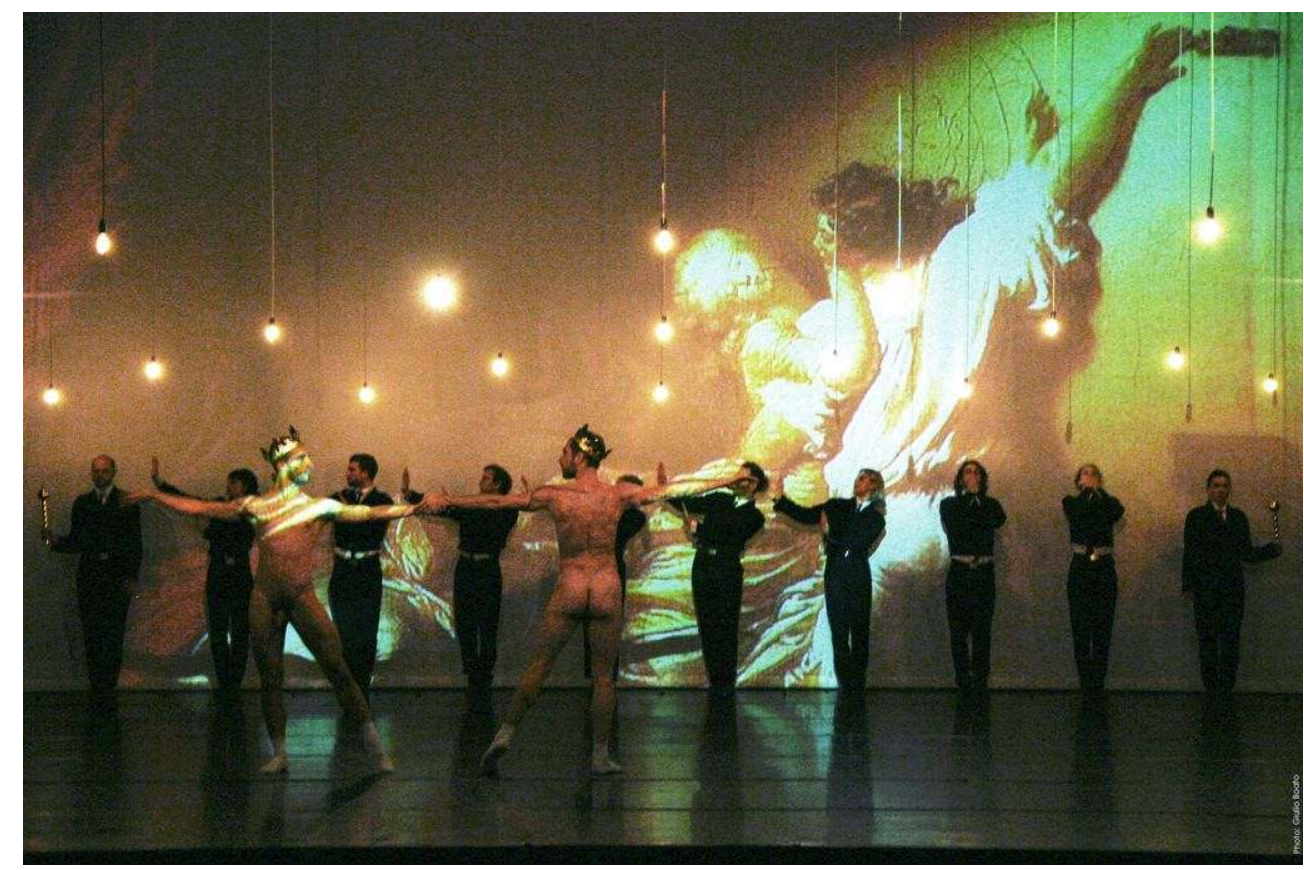

(C) Giulio Boato

Les seuls éléments scénographiques permanents sont les vingt-trois lampes suspendues au plafond - unique source lumineuse du spectacle, si on exclut les découpes externes utilisées en renfort pour certaines scènes - et un écran de projection qui tient lieu de fond de scène sur lequel sont projetées des diapositives de tableaux des $\mathrm{XV}^{\mathrm{e}}$ et $\mathrm{XVIII}{ }^{\mathrm{e}}$ siècles. L'écran fait aussi office de coulisse pour les entrées et sorties des acteurs. 
16 Le spectacle est présenté le 11 Juin 1984 au Théâtre Carlo Goldoni dans le cadre de la Biennale de Venise (durant laquelle Fabre présentera également une série de dessins). Après Venise, le spectacle poursuit sa route pour une tournée mondiale qui durera deux ans. Cette première trilogie de spectacles ${ }^{14}$ inscrit Jan Fabre dans le panorama théâtral international, transformant en quelques années le jeune artiste flamand en une des pépites du théâtre contemporain européen.

\section{Les processus de recréation}

\section{La physicalité fabrienne des performers choisis}

17 Le projet d'une reprise interroge le type de physicalité recherché et la façon dont on peut atteindre l'équilibre délicat entre un retraçage technique de la physicalité des performers d'origine et la physicalité vivante des performers contemporains. Les reprises fabriennes impliquent un double décalage : d'une part, les performers essentiellement amateurs ont fait place à des professionnels auditionnés dans toute l'Europe ; d'autre part, l'exploration collective d'un langage corporel subversif se rapproche aujourd'hui d'une « méthode » de travail en voie de systématisation. Le corps du performer est devenu l'instrument d'une analyse rétrospective de l'esthétique fabrienne. Le rapport entre spontanéité et technicité est ici paradoxal, dans la mesure où Jan Fabre semble quelque peu regretter la spontanéité naturelle des amateurs de l'époque, tout en recherchant une technicité pointue chez ses performers. Pour les nouvelles éditions de C'est du théâtre... et du Pouvoir..., Fabre a en effet fait des auditions à Anvers, Paris, Rome, Athènes et Zagreb, examinant un éventail d'environ mille quatre cents acteurs, danseurs et performers. Il s'est avéré difficile de trouver cette colère chère à Jan Fabre, cette nécessité absolue d'être sur scène: "I'm looking for the real anger, "I want to die for it": they had it naturally those days, they were amateurs in the sense that they were lovers of beauty ${ }^{15}$. " Comment retrouver cet amour de la beauté chez des performers dont la physicalité est notamment conditionnée par une formation professionnelle qui a intégré la grammaire subversive de l'époque?

Il est évident que le niveau technique des performers actuels est nettement supérieur à celui de leurs prédécesseurs, dont la plupart, rappelons-le, n'étaient pas professionnels. Ce décalage s'avère quelque peu problématique lorsqu'il s'agit de définir la nature même des spectacles, en particulier de C'est du Théâtre.... La force de cette pièce se loge dans la présentation aux spectateurs de séquences d'actions quotidiennes, privées de toute signification symbolique et extraites de leur cadre habituel de référence (pratique typiquement dadaïste) et exacerbées à travers la répétition. Toute déformation " dansée » de ces actions finit par modifier le contexte, en changeant le cadre théorique et en détournant le sens de la pièce. Durant les répétitions en 2012, ce risque a été très nettement perçu par Renée Copraij ${ }^{16}$, une des danseuses-actrices fétiches de Fabre qui prend fréquemment en charge la formation des performers. "Today we have to work more on the "daily", affirme Copraij, this is getting clearer in This is theatre, which is about all those daily actions; because it's now becoming more "theatrical", even this piece ${ }^{17}$. "

En observant les jeunes acteurs durant les répétitions, Copraij reconnaît que l'habileté des nouveaux interprètes tend à prévaloir sur les actions qui doivent être accomplies. En 1982, Fabre avait recruté des acteurs non professionnels ${ }^{18}$ qui, au cours des six mois de répétition (et plus d'un an de tournée), avaient acquis les qualités nécessaires pour interpréter le spectacle. La déformation professionnelle des performers actuels tend à 
perturber la simple exécution d'actions qui en elles-mêmes ne demandent pas d'entraînement particulier de danseur ou d'acteur. Le travail final avec les nouveaux performers a nécessité une forme de dépouillement: éliminer tous les tics et automatismes inconscients causés par la pratique professionnelle. C'est $d u$ théâtre... avait été créée pour mettre en branle les conventions théatrales et proposer au public une nouvelle façon de se rapporter à l'événement scénique : une relation entre êtres humains d'égal à égal, pas seulement entre acteurs et spectateurs.

Voilà pourquoi, en deux mois de travail de yoga ${ }^{19}$, Copraij s'est concentrée sur les bases de la discipline, sur les racines de la pratique. Les danseurs entraînés ne rencontraient aucune difficulté à adopter les positions les plus complexes, mais les abordaient d'un point de vue qui n'était pas le bon: le performer travaille sur l'extériorisation, pour montrer; le Yogi travaille sur l'intériorisation, pour être. C'est du théâtre... se veut un spectacle théâtral (il se montre) mais constitue également un accomplissement (il est). La pratique du Yoga constitue donc une formation fructueuse vers l'accomplissement d'actions simples et quotidiennes qui renvoient les spectateurs à leur présent, leur épargnant quelques heures le rythme continu de la société contemporaine :

[in Yoga] What you are practicing is to connect back to yourself, and to take time: a lot of things we do are always projected into the future, and in this way we do exactly what new liberalism and post-fordism wants from us, we do exactly the way things are produced in our society, and I think that as artists and as yogists we should really resist this, we should practice to connect back, take the time, use the time here and now, conserve our authority and be our own boss ${ }^{20}$.

21 Le temps d'acclimatation des nouveaux acteurs a été bien plus bref que pour la première version : la réédition de ce spectacle ne demande pas un processus de création mais plutôt un apprivoisement d'une structure déjà existante. Cela étant, il est à noter que les interprètes ne connaissaient pas les spectacles qu'ils allaient monter : la plupart d'entre eux n'avait jamais vu les vidéos des pièces, et Fabre ne le leur a pas demandé. L'espoir du metteur en scène consistait précisément à avoir entre ses mains de la « matière vierge » à modeler, sans la nécessité de devoir leur expliquer les raisons profondes de son œuvre. Un travail pédagogique a été mené par Fabre, surtout au début des répétitions, au travers, par exemple, de lectures à ses comédiens d'extraits du carnet de jour qu'il rédigea à l'époque de la création des spectacles, afin de donner aux jeunes performers des clés de lecture de son propre travail et de ses opinions sur l'art et le théâtre.

La question de la physicalité s'ancre dans une recherche rétrospective sur la «méthode » fabrienne. Ces dernières années, Fabre travaille à la création d'une ligne directrice pour ses performers, une sorte d'entraînement basé sur des exercices physiques et de réflexion artistique. Le choix de définir ses recherches pédagogiques en une "ligne directrice " dérive du refus de création d'une méthode (comme, par exemple, celles de Stanislavski ou Grotowski) dans la mesure où une méthode, basée sur des actions gymniques concrètes ancrées dans leur temps, devient rapidement obsolète. Pour Fabre, la «ligne directrice » peut en revanche persister, en reflétant la dimension contingente et en affirmant la condition même du performer qui se perçoit comme tel. Repensant à ses premiers spectacles, Fabre s'est rendu compte que, dans cette « simplexity 》 (simplicity + complexity) à la base de ses premiers spectacles, résident tous les exercices qu'il a repris successivement pour transformer ses nouveaux danseurs et acteurs en «Guerriers de la Beauté21. » Présenter à nouveau Le pouvoir... et C'est du théâtre... signifie, pour le metteur en scène, se donner l'opportunité d'étudier avec du recul les origines de sa propre poétique, ramenant à la vie l'essence initiale de laquelle toutes ses productions successives se sont 
nourries. Ramener à la vie implique bien évidemment de dépasser un point de vue archiviste afin d'adopter une posture modulaire, ouverte aux réalités de notre temps. Ici réside sans doute toute la délicatesse de l'équilibre entre une systématisation et une flexibilité qui permet la pertinence au fil du temps.

Cette recherche d'une définition de sa ligne de conduite s'inscrit par ailleurs dans une volonté de former une nouvelle génération de performers, en vue d'un spectaclemarathon prévu pour 2015.

\section{À la recherche d'une structure précise} année de représentation, metteur en scène et acteurs, encore inexpérimentés et en continue recherche de nouvelles solutions, essayèrent plusieurs variantes. De plus, Fabre interchange souvent les rôles de ses acteurs, parfois pendant la tournée, peu avant d'entrer en scène. Le metteur en scène exige que chaque acteur connaisse tout le spectacle et puisse endosser ainsi n'importe quel rôle : «I change people to make them alert, awake, to not step into the trap of automatism ${ }^{24}$.

Les deux reprises ont été préparées parallèlement, en huit semaines, sur base des vidéos d'archive et des notes de mise en scène originales qui, en plus des textes, contiennent les descriptions détaillées des mouvements.

Pour Amelia Jones ${ }^{22}$, ce n'est que depuis récemment que l'on s'interroge sur la fiabilité des archives à la base des reprises - qui n'ont plus désormais valeur de preuve - et sur la façon dont ces documents réduisent la matière vivante originale à des objets visuels échangeables. Pour la chercheuse, dans le meilleur des cas, les reprises travaillent sur le paradoxe par lequel la documentation est utilisée pour créer une reprise... qui devient elle-même un objet de documentation. Si la question des limites de la documentation est pleinement assumée par Fabre, nous verrons que la question de la transformation du spectacle en objet de documentation est plus problématique.

Dans le cas des reprises fabriennes, le matériel d'archive a été abordé de façon distanciée. Les limites assumées de la documentation s'expliquent premièrement par la forme des spectacles du metteur en scène; aucun spectacle de Fabre n'a de version définitive avant son ultime représentation : "I teach my company : the last performance will be the first one ${ }^{23}$. " La structure des spectacles n'est jamais totalement figée; l'évolution de l'œuvre dans le temps demande certaines modifications, améliorations, recadrages.

L'instabilité de la forme est condition originelle pour C'est du théâtre... : durant la première Pour ces raisons, la vidéo d'archive et la mise en scène elle-même ne sont abordées que comme deux traces d'une constellation de petites variantes éphémères, dissoutes en même temps que la sortie des spectateurs, à la fin de chaque représentation.

Une grande partie du travail a été faite également sur la base de souvenirs personnels du metteur en scène et de son inséparable assistante, Miet Martens, qui intégra la compagnie un mois avant la tournée du Pouvoir des folies théâtrales, et en suivit les autres soixante représentations par-delà le monde. Pour faire face aux limites de la documentation et de la mémoire, partiale et incertaine, Fabre et son équipe ont ajusté quelques détails durant le processus de répétition. Ils ont ajouté quelques textes au Pouvoir..., modifié quelques traductions (le texte est toujours multilingue) en relation avec les connaissances linguistiques des nouveaux performers et proposé quelques légères variantes pour C'est $d u$ théâtre.... Dans ce spectacle particulièrement, la partition scénique n’a pas été calquée 
scrupuleusement sur l'originale: si la structure de base demeure, on constate le remaniement de certaines actions internes, le changement de petits détails pour inspirer les acteurs afin de donner un nouveau souffle. La version 2012 de C'est du théâtre... prévoit un acteur supplémentaire par rapport au cast original: pour ne rien perdre des précieuses différences des qualités d'interprétation des nouveaux performers, le rôle d'une des premières actrices (Els Deceukelier, une des muses du metteur en scène) a été divisé entre deux nouvelles danseuses ${ }^{25}$. Les acteurs ne sont jamais plus de huit en scène comme dans la version originale - le dernier tableau excepté, où sont présents les neuf performers.

30 La forme n'est donc pas strictement figée, et se concrétise par une présentation scénique qui permet une certaine appropriation au performer contemporain (voir point suivant).

Nombreux sont les chercheurs qui insistent sur la nécessité d'ajuster l'œuvre originale au contexte contemporain, sans quoi, selon Morgan par exemple, la reprise risque de se transformer en simulation peu convaincante, voire en "faux volontaire» ou en " véritable kistch ${ }^{26}$ ».

Fabre ne veut bien entendu pas proposer une pâle copie de ses créations et ne fige pas la représentation en un cliché immuable ; les modifications - sommaires - apportées ne font cependant pas entrer en dialogue l'œuvre originale et la reprise. Elles ne concrétisent pas une volonté de moduler le spectacle et de souligner combien la performance passée diffère de celle présentée aujourd'hui.

33 A titre de contre-exemple, Christel Stalpaert ${ }^{27}$ a montré combien la reprise des solos de Mary Wigman par Fabian Barba met en évidence le fossé corporel entre l'œuvre-source et la reprise plutôt que de tenter de les dissimuler. Stalpaert rappelle l'allégorie du chiffonnier proposée par Walter Benjamin et appliquée à l'histoire du théâtre par Frédéric Le Roy, selon laquelle le créateur de la reprise préfère montrer « les chiffons » de l'histoire plutôt que sa dimension monumentale. Les décalages soulignés par Barba subvertissent notre approche de l'œuvre iconique. En manipulant nos horizons d'attente, il interroge notre regard sur l'œuvre appartenant désormais au répertoire. La double prétention des artistes-repreneurs mentionnée précédemment peut expliquer la posture fabrienne: le décalage ne serait pas à souligner dans la mesure où ses classiques de l'avant-garde parleraient toujours au public d'aujourd'hui. La relative flexibilité de la forme favoriserait cette transposition. Au demeurant, les modifications apparaissent comme une actualisation de la forme originale en fonction des conditions de reproduction actuelles (spécificités des performers, etc.) plutôt que comme un rapport dialectique entre les deux versions. Interviewée à ce sujet, Miet Martens répond:

We tried to do it as pure as it used to be, not immediately similar but with the same ideas, not bringing more things in, not translating it into 2012. [...] We wanted to add more references to the text, we didn't like the 4th scene of The power as it was in the video. If you have the same director, he is allowed to change that, for $\mathrm{me}^{28}$.

Fabre assume cette position peu dialectique; son ambition résidait dans la réapparition d'œuvres passées pour en mesurer l'efficacité aujourd'hui. Ces reprises participent à la mise en système de son parcours artistique. D'ailleurs, son dernier spectacle Tragedy of $a$ Friendship (2013) comprend de nombreuses résonances avec ses premiers spectacles. Au demeurant, l'opération de Fabre constitue un travail sur soi-même, en fonction d'une réévaluation de ses premières productions au service de ses futures créations. Fabre témoigne explicitement de ce désir de retour à l'origine: "I'm happy that I did those two 
reenactments because I went so far away over these 30 years from that kind of work that it is for me now refreshing and almost new to see this again and use some of these rules again $»^{29}$.

\section{L'authenticité de la présence}

Le mode opératoire du metteur en scène - varier les détails des actions en conservant la structure du spectacle - trouve un parallèle dans la façon de jouer de ses acteurs: l'organisation des mouvements est extrêmement précise; l'espace est mesuré au centimètre et chaque position est signalée au sol par un adhésif (noir, de façon à ce qu'il soit invisible pour les spectateurs). Cette structure rigide est créée pour être animée par la personnalité de l'acteur, par son inventivité et sa vitalité. Chaque soir, les acteurs doivent apporter de minimes variantes à leurs gestes, réinventer leurs actions, retrouver leur raison d'être et leur nécessité d'être sur scène.

Pour Marleen Wynants ${ }^{30}$, Jan Fabre nous propose une «confrontation directe » avec le « corps humain non médiatisé »; la mise en fragilité du performer sur le plateau permet l'apparition de l'imprévu. La répétition des actions est une méthode utilisée par le metteur en scène afin d'éprouver "l'énergie indomptable ». La répétition engendre une progression ; chaque niveau atteint est un nouveau départ pour parvenir à cette énergie de l'épuisement, encore et encore. Le spectacle stimulerait ainsi la présence authentique. Pour Fabre, le caractère "réel» de la performance est aujourd'hui accentué par l'omniprésence des sons et images médiatisés dans notre vie quotidienne (voir point suivant).

D'aucuns aujourd'hui relativisent l'authenticité de la présence scénique. Depuis l'approche kantienne de l'insaisissabilité du réel, dont notre connaissance est invariablement tributaire de notre subjectivité, la présence apparait pleinement dans sa volatilité. Amelia Jones ${ }^{31}$ souligne la contradiction inhérente à de nombreuses reprises, à savoir que l'on présente un événement live au spectateur, se déployant dans l'ici et maintenant, tout en sachant que cette immuabilité est impossible puisque l'événement a déjà eu lieu. Tout événement live est insaisissable; sa finitude se marque au fur et à mesure de son déroulement. La présence ne peut être perçue dans son authenticité. Par conséquent, la prétention de certaines reprises, comme le cycle de performances de Marina Abramović au MoMA en 2010, à atteindre cette présence authentique se révèle irrémédiablement utopique. Au demeurant, Jan Fabre ne cherche pas à retrouver l'authenticité d'une présence révélée trente ans plus tôt ; les performers ont changé et teintent les reprises d'une relative personnalisation. Par contre, les innombrables répétitions des actions scéniques et des fragments audiovisuels inscrites dans C'est $d u$ théâtre... mettent précisément en évidence combien la présence se construit en tant qu'objet de représentation. La reprise de ce théâtre performatif souligne paradoxalement combien la recherche d'une présence originale authentique constitue un horizon inatteignable. La thématique-même de la répétition, ancrée au cœur du spectacle, fait apparaitre en filigrane cette hypothèse. Roger Copeland va dans ce sens lorsqu'il nous rappelle que les discours rapportant une présence « pure » ou « préverbale » comme chez Artaud, etc., ont été particulièrement remis en question ces dernières années : « Il est loin d'être clair que le théâtre ait un jour été le dépositaire unique ou essentiel de la présence. [...] La présence, la pureté, l'instantanéité, l'anti-illusionnisme ont tous battu en retraite ${ }^{32}$ . " 
Alors que la répétition est explorée dans le but d'atteindre l'énergie indomptable de l'épuisement et de parvenir ainsi au « réel » dégagé de toute représentation, elle souligne, par un retour de manivelle, la médiation de la présence et l'impossibilité de la présence authentique.

39 A priori, Fabre semble bien conscient de ce paradoxe; on peut particulièrement s'en apercevoir dans Le pouvoir..., où il joue perpétuellement entre la réalité et la fiction. On peut considérer que Le pouvoir... se pose comme une réponse à C'est du théâtre... : si dans ce dernier il voulait, effectivement, atteindre le réel par la répétition, dans le spectacle suivant il prend pleinement conscience de l'impossibilité de "performer le réel», et s'amuse à frapper les spectateurs avec la démonstration de la persistance de la fiction dans le « théâtre ».

\section{En dialogue avec le public d'aujourd'hui}

Morgan ${ }^{33}$ cristallise le positionnement des études théoriques des reprises lorsqu'il insiste sur l'importance de débloquer la connexion patrimoniale à l'Histoire afin de transcender l'époque de création de l'œuvre originale. Pour ce faire, il est indispensable d'ouvrir le spectacle à d'autres niveaux - psychologiques, narratifs et interactifs.

Pour Fabre, la portée des spectacles de ses débuts sur le public d'aujourd'hui s'ancre particulièrement dans deux décalages par rapport au contexte contemporain : d'une part, ces reprises qui font la part belle à la corporalité des performers se confrontent à la médiatisation toujours plus importante de notre vie quotidienne. D'autre part, la durée extra-ordinaire de C'est du Théâtre... (huit heures) et du Pouvoir... (cinq heures) les place en contrepoint des habitudes culturelles : comment se confronter à la longue durée d'une production, quand désormais chaque évènement est de courte durée ? Fabre permettrait ainsi à la temporalité de faire son office : « Le spectacle dure huit heures, mais ce n'est en aucun cas un marathon. Cette durée, c'est pour laisser aux choses le temps d'avenir, de laisser se faire le processus chronologique ${ }^{34}$. » Pour Fabre, prendre le temps de s'asseoir et de regarder durant huit heures une séquence scénique ininterrompue (potentiellement infinie en raison d'une issue qui ne s'annonce pas) est une expérience déstabilisante pour un public habitué aux quatre-vingt-dix minutes standard: " you have to cool down, to go to the details : time becomes an architectural element ${ }^{35}$. "

La durée du spectacle et l'importance des technologies numériques sont également deux questions centrales pour Bob Wilson lorsqu'il reprend Einstein on the Beach (cinq heures). Alors que le développement des technologies numériques est une donnée à bouleverser pour Fabre, il constitue un contexte plutôt favorable au spectacle pour Wilson : « Rien n'a changé mais maintenant nos yeux et nos oreilles sont prêts à voir et à entendre. [...] Le public a changé, donc l'œuvre sera forcément perçue de façon différente. Le public d'aujourd'hui a une expérience beaucoup plus grande de l'informatique et de l'électronique. La musique électronique est partout. La génération actuelle se sent probablement plus proche de la façon de penser à l'œuvre dans Einstein on the Beach que ne l'était le public à sa création ${ }^{36}$.»

Les deux artistes s'interrogent par contre tous deux sur la capacité du public à profiter des cinq ou huit heures du spectacle.

Par ailleurs, Fabre inscrit cette subversion de la temporalité actuelle dans un discours s'opposant aux pratiques commerciales de la culture. Pour Fabre, les deux spectacles sont 
" against the complete theatre system which asks to not make it difficult, to be entertaining, to be sure that you get good reviews ${ }^{37} »$. Cela demanderait donc un effort supplémentaire et une prise de risque de la part des programmateurs et directeurs de théâtre par rapport à un éventuel refus du public. La nuit blanche de C'est $d u$ théâtre... est une expérience éprouvante pour les spectateurs : lors de la première à Vienne en 2012, toutes les places étaient vendues, mais des 350 personnes entrées à 21 heures le 21 juillet, un peu moins de la moitié d'entre elles sont restées jusqu'à 5 heures du matin ${ }^{38}$.

On peut s'étonner du positionnement de Fabre, qui semble ici s'opposer à un système auquel il n'appartient pas directement. Doit-on en effet considérer que les structures accueillant les pratiques spectaculaires contemporaines visent réellement le divertissement de leur public? S'il y avait une telle subversion il y a trente ans, celle-ci ne ressemble en rien au contexte actuel. De plus, Fabre semble ici occulter la dynamique commerciale dont il est à l'origine : la renommée de l'artiste fait résolument vendre, et lui permettrait de "tirer sur la ficelle, selon ses détracteurs " ${ }^{39}$. Le positionnement socioéconomique de ces reprises, qui dépasse la position de l'artiste, n'est donc pas dénué de contradictions.

Pour Fabre, ces spectacles actualisent leur vitalité en bouleversant les habitudes numériques et temporelles du public aujourd'hui. Pour autant, que viennent précisément voir ces spectateurs? Qu'applaudissent les spectateurs au terme de la représentation? Qu'est-ce qui a décidé les autres de quitter la salle avant la fin? Sarah Vankersschaever ${ }^{40}$ souligne le contraste entre les réactions dans les années quatre-vingt, où certains spectateurs voulaient être remboursés, voire frapper Fabre, et les réactions aujourd'hui, où il est à craindre que les spectateurs rentrent bravement à la maison, sans que la soirée ne donne naissance à un débat engagé. "Sommes-nous devenus trop dociles? Ou sommes-nous plus habitués que dans les années quatre-vingt? Ou n'osons-nous pas dire tout haut que nous n'apprécions pas un classique?»

Ceci dit, il importe de nuancer quelque peu l'hypothèse d'une passivité du public contemporain. Lors de la première du Pouvoir des folies théâtrales en 2012, il y a eu énormément de hurlements dégoûtés émanant de spectateurs, lorsque ceux-ci croyaient que les acteurs écrasaient des grenouilles sur scène.

Klaas Tindemans ${ }^{41}$, présent dans la salle lors de la présentation de C'est du théâtre... en 1982, souligne l'absence de cadre à l'époque, ce qui explique les réactions très négatives, tant dans la presse que parmi certains spectateurs. Aujourd'hui, la réputation précédant la représentation, le public fait confiance au jugement d'une communauté de goût qui a canonisé certains spectacles, devenus des icônes du répertoire. Le risque est dès lors grand de développer chez les spectateurs une curiosité et un horizon d'attentes rétrospectifs. Les spectateurs viennent, restent ou quittent la salle, comme trente ans auparavant, mais pour des raisons parfois bien différentes.

\section{Au risque de la reprise}

49 Le phénomène international des reprises touche aussi la Belgique néerlandophone. Les reprises par Jan Fabre de deux de ses premiers spectacles - C'est du Théâtre comme c'était à espérer et à prévoir (1982) et Le Pouvoir des folies théâtrales (1984) ont constitué des moments-clés de la saison théâtrale 2012-2013. Ceci étant, elles comprennent certains paradoxes et contradictions, ce qui met en évidence la délicate entreprise que constitue la 
recréation d'un spectacle, d'autant plus s'il est devenu iconique. La question porte d'une part sur les processus de création: la physicalité des performers et le rapport à la documentation sont des problématiques essentielles. Jan Fabre explore un équilibre fragile entre une structure millimétrée et des espaces d'expression personnelle pour ces nouveaux performers. D'autre part se pose la question du dialogue entre l'œuvre, le contexte de départ et celui d'arrivée, et les spectateurs d'aujourd'hui. Si l'œuvre s'actualise comme un contrepied de nos pratiques numériques quotidiennes, sa reprise vient conforter son statut de canon, appartenant au répertoire, et souligne en filigrane combien ces œuvres patrimonialisées peuvent constituer un certain levier de fonds et d'attraction.

Pour Fabre, la mise en test de l'efficacité de ces œuvres par leurs reprises s'est révélée positive :

«When last year I saw again the first scenes of The power..., I was touched. I think the productions are now stronger and better than thirty years ago. The pieces are still radical ${ }^{42}$."

51 Le titre de C'est du Théâtre comme c'était à espérer et à prévoir prend néanmoins aujourd'hui une nouvelle couleur: trente ans plus tard, ce théatre performatif subversif est quelque peu devenu du théâtre à espérer et à prévoir. La complexité de la pratique de la reprise nous oblige à en tenir compte, sans quoi aucun dialogue entre l'œuvre originale et l'œuvre aujourd'hui n'est réellement possible.

\section{BIBLIOGRAPHIE}

ARVERS, Fabienne, LE TANNEUR, Hugo, NOISETTE, Philippe, « Einstein on the Beach, une œuvre visionnaire et unique », Les Inrocks, 16 mars 2012

COPELAND, Roger, «The Presence of Mediation », The Drama Review, 1990, vol. 34, n 4, pp. 28-44 DUPLAT, Guy, « Le Fabre pur jus, 30 ans de radicalité », La Libre Belgique, 27 juin 2012 JONES, Amelia, «"The Artist is present.” Artistic Re-enactments and the Impossibility of Presence ", The Drama Review, 2011, vol. 55, n 1, pp. 16-45

LAURENT, Anne, « Jan Fabre toute une nuit », Libération, 24 octobre 1983

MAURIN, Frédéric, « Sous la cendre, les braises : actualité et usages des années 1960-1970 », Théâtre/Public, janvier-mars 2012, n² 203, pp. 16-23

MORGAN, Robert C., « Thoughts on Re-Performance, Experience and Activism », PAJ: A Journal of Performance and Art, 2010, vol. 32, $\mathrm{n}^{\circ}$ 3, pp. 1-15

MULDERS, Sofie, « 80 is prachtig, ook op het podium », De Morgen, 14 février 2013

SCHECHNER, Richard, «L'avant-garde, la niche-garde et la "Performance Theory" » dans HELBo, André, BOUKo, Catherine et VERLINDEN, Elodie (dir.), Performances et savoirs, Bruxelles, De Boeck, 2011, pp. 15-36 
STALPAERT, Christel, « Reenacting Modernity: Fabian Barba's A Mary Wigman Dance Evening (2009) », Dance Research Journal, vol. 43, n² 1, été 2011, pp. 90-95

THIBAUDAT, Jean-Pierre, « Huit heures sans entracte de théâtre tout cru », Libération, 3 mai 1983

VAN DEN DRIES, Luk, Corpus Jan Fabre. Observations sur un processus de création, Paris, L'Arche, 2005

VANKERSSCHAEVER, Sarah, « Fabre is niet langer brutaal », De Standaard, 28 septembre 2012

WYNANTS, Jean-Marie, « Jan Fabre, classique et iconoclaste », Le Soir, 22 octobre 2012

WYNANTS, Marleen, « Het complete theater van Jan Fabre in twaalf uur », distribué les soirs de représentation au Kaaitheater.

\section{NOTES}

1. Ce spectacle, créé en 1976 lors du festival d'Avignon avait déjà été repris en 1984 et 1992.

2. Richard, Schechner, "L'avant-garde, la niche-garde et la "Performance Theory" » dans André, Helbo, Catherine, Bouko et Elodie,Verlinden (dir.), Performances et savoirs, Bruxelles, De Boeck, 2011, p. 17.

3. Ibidem.

4. Erwin, Jans cité dans Sofie, Mulders, " 80 is prachtig, ook op het podium », De Morgen, 14 février 2013.

5. Jan, Fabre cité dans Guy, Duplat, « Le Fabre pur jus, 30 ans de radicalité », La Libre Belgique, 27 juin 2012.

6. Richard, Schechner, "L'avant-garde, la niche-garde et la "Performance Theory" », art. cit., p. 25.

7. Frédéric, Maurin, "Sous la cendre, les braises : actualité et usages des années 1960-1970 ", Théâtre/Public, janvier-mars 2012, n²03, p. 17.

8. Ibidem.

9. David, Savran, « Death of the avant-garde ", The Drama Review, 2005, Vol. 49, n³, p.36, cité dans Richard, Schechner, "L'avant-garde, la niche-garde et la "Performance Theory" ", art. cit., pp. 17-18.

10. Jan, Fabre, cité dans Anne, Laurent, «Jan Fabre toute une nuit », Libération, 24 octobre 1983.

11. "Citations, voire présentations: un entretien radiophonique avec Marcel Duchamp ", (Audio Art Magazine, 1976) est diffusé à différents moments du spectacle.

12. Jean-Pierre, Thibaudat, " Huit heures sans entracte de théâtre tout cru ", Libération, 3 mai 1983.

13. "Quand je dois choisir entre la colère et la technique, je choisirai toujours celui qui a de la colère.» Entretien entre Jan Fabre et Giulio Boato, Anvers, 8 juin 2012, enregistrement sonore inédit. Vu la grande quantité d'informations extraites de ce document dans le présent article, on considère que chaque citation en langue anglaise, dont n'est pas spécifiée la source, provient de ce même entretien.

14. La première production de Jan Fabre est Theater geschreven met een $K$ is een kater en 1980.

15. " Je cherche la véritable colère, "Je veux mourir pour cela" : ils l'avaient de façon naturelle à cette époque, ils étaient des amateurs dans le sens qu'ils étaient des amoureux de la beauté. »

16. Entrée dans la compagnie en 1987 pour le ballet Das Glas im Kopf wird vom Glas - The dance sections, Copraij a travaillé comme performer de Fabre jusqu'en 1998 (The fin comes a little bit earlier this century - But business as usual). Depuis 2002 (Swan Lake) elle est devenue assistante à la dramaturgie et entraîneuse des performers pour de nombreux spectacles de Fabre. 
17. « Aujourd'hui, nous devons travailler davantage sur le "quotidien" » [...] « Cela devient plus net avec C'est $d u$ Théâtre, qui concerne toutes ces actions quotidiennes, parce que c'est maintenant en train de devenir plus "théâtral", même cette pièce. " Entretien entre Renée Copraij et Giulio Boato, Anvers, 22 juin 2012, enregistrement sonore inédit.

18. Par souci de fidélité, l'actrice professionnelle Rena Vets était présente lors des répétitions. Elle avait à l'époque un peu plus de la vingtaine.

19. Discipline intégrée à l'entraînement quotidien des performers, avec le kendo et les cours de Fabre. Cette pratique n'était pas présente en 1982-4.

20. « [En yoga], ta pratique est de te reconnecter avec toi-même, et de prendre le temps : beaucoup de choses que l'on fait sont toujours projetées dans le futur, et de cette façon nous faisons exactement ce que le nouveau libéralisme et le post-fordisme veulent de nous, nous procédons exactement de la manière dont les choses sont produites dans notre société, et je pense qu'en tant qu'artistes et que yogistes, nous devrions vraiment résister à cela, nous devrions pratiquer afin de nous reconnecter, de prendre le temps, d'utiliser le temps ici et maintenant, de conserver notre autorité et être notre propre maître. " Entretien entre Renée Copraij et Giulio Boato, op. cit.

21. Jan, Fabre : «Mes acteurs et mes danseurs sont des guerriers de la beauté qui, dans l'univers de mes pièces, ont le courage de vivre dangereusement et de prendre le risque de faillir ou de triompher ", cité dans Luk, Van den Dries, Corpus Jan Fabre. Observations sur un processus de création , Paris, L'Arche, 2005, p. 343. Selon Fabre, le performer devient un guerrier, celui qui met en jeu sa propre vie pour vaincre le combat en lequel il croit : la lutte entre le théâtre et la vie, entre acteurs et spectateurs, entre la société et la nature. Mais aussi la lutte intérieure à l'acteur, qui est un organique champ de bataille entre corps et esprit, entre discipline et instinct.

22. Amelia, Jones, " "The Artist is present." Artistic Re-enactments and the Impossibility of Presence », The Drama Review, 2011, Vol. 55, n¹, pp. 21-24.

23. " j'enseigne à ma compagnie : la dernière performance sera la première. »

24. «Je change les gens pour les rendre alertes, éveillés, pour ne pas tomber dans le piège de l'automatisme. »

25. Ces deux interprètes sont très différentes l'une de l'autre : d'une part par leur formation initiale (l'une est danseuse classique, l'autre danseuse contemporaine) et d'autre part par leur différence physique. Après plusieurs semaines de tentatives, Fabre a décidé de garder les deux filles, car chacune s'était appropriée certaines scènes ; en choisir une seule aurait affaibli le spectacle : «I had to bow down to beauty », nous a-t-il avoué en souriant.

26. Robert C., Morgan, "Thoughts on Re-Performance, Experience and Activism », PAJ: A Journal of Performance and Art, 2010, Vol. 32, n³, p. 2.

27. Christel, Stalpaert, « Reenacting Modernity : Fabian Barba's A Mary Wigman Dance Evening (2009) ", Dance Research Journal, Vol. 43, n¹, été 2011, pp. 90-95.

28. "Nous essayons de le faire de la façon aussi pure que cela a pu être, pas immédiatement similaire mais avec la même idée, ne pas apporter plus de choses, ne pas le traduire pour 2012. [... ] Nous voulions ajouter plus de références au texte, nous n'aimions pas la 4e scène du Pouvoir comme elle était dans la vidéo. Si tu as le même metteur en scène, il est autorisé à changer cela, selon moi. » Entretien entre Miet Martens et Giulio Boato, Anvers, 3 juillet 2012, enregistrement sonore inédit.

29. «Je suis heureux d'avoir fait ces deux reprises parce que j'ai été si loin de ce type de travail ces trente dernières années que c'est maintenant rafraîchissant pour moi et presque nouveau de le voir à nouveau et d'utiliser à nouveau certaines de ces règles. "

30. Marleen, Wynants, « Het complete theater van Jan Fabre in twaalf uur », distribué les soirs de représentation au Kaaitheater.

31. Amelia, Jones, " "The Artist is present." Artistic Re-enactments and the Impossibility of Presence », art. cit., pp. 16-45. 
32. Roger, Copeland, «The Presence of Mediation ", The Drama Review, 1990, Vol. 34, n4 , p. 39.

33. Robert C., Morgan, "Thoughts on Re-Performance, Experience and Activism », art. cit., pp. 2-5.

34. Jan, Fabre cité dans Anne, Laurent, «Jan Fabre toute une nuit », art. cit.

35. « Tu dois te détendre, aller dans les détails : le temps devient un élément architectural. »

36. Citation de John Cage fréquemment rappelée par Robert Wilson, dans Fabienne, Arvers, Hugo,

Le Tanneur et Philippe, Noisette, "Einstein on the Beach, une œuvre visionnaire et unique ", Les Inrocks, 16 mars 2012.

37. " contre la totalité du système théâtral qui nous demande de ne pas faire de choses difficiles, d'être divertissant, de s'assurer d'obtenir de bonnes critiques. "

38. Dépassant cela dit les attentes du metteur en scène, qui avant d'entrer en scène avait préparé ces acteurs au pire, rappelant qu'en 1982 ils avaient conclu plusieurs représentations avec dix personnes en salle.

39. Jean-Marie, Wynants, "Jan Fabre, classique et iconoclaste », Le Soir, 22 octobre 2012.

40. Sarah, Vankersschaever, " Fabre is niet langer brutaal », De Standaard, 28 septembre 2012.

41. Klaas, Tindemans, cité dans Sarah ,Vankersschaever, « Fabre in niet langer brutaal », art. cit.

42. «Quand j'ai revu l'an passé les premières scènes du Pouvoir..., j'étais touché. Je pense que les productions sont maintenant plus fortes qu'il y a trente ans. Les pièces sont toujours radicales. »

\section{RÉSUMÉS}

Les reprises de spectacles majeurs de ces trente ou quarante dernières années s'invitent de plus en plus dans les saisons théâtrales européennes. Réalisées le plus souvent par l'artiste-créateur lui-même, ces reprises témoignent d'une double posture énonciative: pour l'artiste, l'enjeu consiste dans la possibilité d'offrir le spectacle aux nouvelles générations et de vivifier sa capacité à toucher les spectateurs d'aujourd'hui, tout en consolidant, de façon explicitement assumée ou non, son spectacle dans le patrimoine spectaculaire. Dans cet article, nous proposons d'examiner la pratique de la reprise au travers de l'analyse des reprises de C'est du théâtre comme c'était à espérer et à prévoir (1982) et du Pouvoir des folies théâtrales (1984) créées par Jan Fabre. Tant la posture de l'artiste que les attentes spectatorielles apparaissent dans leur complexité.

\section{INDEX}

Mots-clés : C'est du théâtre comme c'était à espérer et à prévoir, Fabre (Jan), patrimoine, reprise, subversion

\section{AUTEURS}

\section{GIULIO BOATO}

Giulio Boato, diplômé en études théâtrales à l'Université de Venise (IUAV) et à l'Université de Bologne, s'occupe de mise en scène d'un point de vue tant théorique que pratique. Il a écrit sur les démarches de Robert Lepage et Jan Fabre pour les revues Antropologia e Teatro, Revista 
Brasileira de Estudos da Presença et Alfabeta2. Il collabore avec plusieurs structures européennes : Troubleyn/Jan Fabre (Anvers), Compagnies des Indes (Paris), Emilia Romagna Teatri Fondazione (Modena, Alliance Française de Venise).

\section{CATHERINE BOUKO}

Catherine Bouko est maître de conférences au sein de la filière Spectacle vivant de l'Université Libre de Bruxelles. Ses travaux portent essentiellement sur la réception des spectacles interartistiques, immersifs et intermédiaux contemporains. Elle a notamment publié l'ouvrage Théâtre et Réception. Le Spectateur postdramatique aux Editions Peter Lang en 2010 et a codirigé l'ouvrage Corps et Immersion en 2012 (L'Harmattan). 